\title{
Conversion-driven freeze-out: Dark matter genesis beyond the WIMP paradigm
}

\author{
Mathias Garny, ${ }^{a}$ Jan Heisig ${ }^{* b}$ Marco Hufnagel, ${ }^{c}$ Benedikt Lülf $^{d}$ and Stefan Vogl ${ }^{e}$ \\ ${ }^{a}$ Technische Universität München, James-Franck-Str. 1, 85748 Garching, Germany \\ ${ }^{b}$ Centre for Cosmology, Particle Physics and Phenomenology (CP3), Université catholique de \\ Louvain, Chemin du Cyclotron 2, B-1348 Louvain-la-Neuve, Belgium \\ ${ }^{c}$ DESY, Notkestraße 85, D-22607 Hamburg, Germany \\ ${ }^{d}$ Institute for Theoretical Particle Physics and Cosmology, RWTH Aachen University, \\ Sommerfeldstr. 16, 52056 Aachen, Germany \\ ${ }^{e}$ Max-Planck-Institut für Kernphysik, Saupfercheckweg 1, 69117 Heidelberg, Germany \\ E-mail: mathias.garnyetum.de, jan.heisigeuclouvain.be, \\ marco.hufnagelddesy.de, benedikt.luelf@gmx.de, stefan.vogl@mpi-hd.mpg.de
}

\begin{abstract}
We consider dark matter (DM) with very weak couplings to the standard model (SM), such that its self-annihilation cross section is much smaller than the canonical one, $\langle\sigma v\rangle_{\chi \chi} \ll 10^{-26} \mathrm{~cm}^{3} / \mathrm{s}$. In this case DM self-annihilation is negligible for the dynamics of freeze-out and DM dilution is solely driven by efficient annihilation of heavier accompanying dark sector particles provided that DM maintains chemical equilibrium with the dark sector. This chemical equilibrium is established by conversion processes which require much smaller couplings to be efficient than annihilation. The chemical decoupling of DM from the SM can either be initiated by the freeze-out of annihilation, resembling a co-annihilation scenario, or of conversion processes, leading to the scenario of conversion-driven freeze-out. We focus on the latter and discuss its distinct phenomenology.
\end{abstract}

Corfu Summer Institute 2018 "School and Workshops on Elementary Particle Physics and Gravity" (CORFU2018)

31 August - 28 September, 2018

Corfu, Greece

${ }^{*}$ Speaker. 


\section{Introduction}

A wide range of cosmological and astrophysical observations require the extension of the standard model (SM) of particle physics by a (cold) dark matter (DM) candidate, see e.g. [1] for a review on its evidence. Its abundance in the Universe today can be elegantly explained by a thermal relic from early Universe dynamics. Among such scenarios, thermal freeze-out is one of the simplest and most attractive ones. In this case DM is assumed to be in thermal contact in the very early Universe and decouples once the temperature drops significantly below the DM mass. Appealing features of this mechanism are that the resulting abundance is independent of the not very-well constrained physics prior to freeze-out (e.g. inflation and reheating) and that DM is guaranteed to be sufficiently cold to allow for successful structure formation. In the simplest freezeout scenario the break-down of DM self-annihilation initiates the chemical decoupling of DM from the SM. In this case, imposing the relic abundance to match the observed value, $\Omega_{\mathrm{CDM}} h^{2} \simeq 0.12$, requires the respective thermally averaged annihilation cross section to be of the order of $\langle\sigma v\rangle_{\chi \chi} \sim$ $g_{\chi}^{4} / m_{\chi}^{2} \sim 10^{-9} \mathrm{GeV}^{-2}$. This relation singles out an allowed band in the plane spanned by the DM mass, $m_{\chi}$, and the characteristic DM-SM coupling strength, $g_{\chi}$. It contains the weakly interacting massive particle (WIMP) as a special case, $m_{\chi} \sim v_{\mathrm{EW}}, g_{\chi} \sim g_{\mathrm{EW}}$.

A variety of ongoing experiments probe large portions of this band. However, most searches are subject to a large model-dependence. For models with dominant $s$-wave annihilation the most direct test of the self-annihilating nature of DM is provided by indirect detection experiments. Masses below a few $\mathrm{GeV}$ are excluded by limits from the non-observation of effects of energy injection during recombination in the cosmic microwave background [2]. These limits are rather independent of the actual annihilation process and do not contain uncertainties from the DM density profile. For larger masses limits from Fermi-LAT dwarfs [3] and eventually from cosmic-ray antiprotons [4] constrain the above scenario, reaching up to $m_{\chi} \simeq 500 \mathrm{GeV}$ for non-leptonic annihilation channels.

These results and a variety of additional experimental constraints narrow down the allowed parameter space of the simplest models of thermal relics. Hence it is interesting to consider alternative thermal scenarios that do not just evade these bounds but could potentially point to novel and unexplored signatures of DM. In this article we consider scenarios where the self-annihilation of DM is not responsible for the dynamics of freeze-out. In fact, we assume it to be negligible $\langle\sigma v\rangle_{\chi \chi} \ll 10^{-9} \mathrm{GeV}^{-2}$. This can be realized once a second, slightly ${ }^{1}$ heavier particle is contained in the dark sector and drives the dilution of the dark sector abundances as it can be the case in the coannihilation scenario [6]. In this scenario conversion processes within the dark sector are assumed to be efficient thus maintaining chemical equilibrium among dark sector particles. Consequently, chemical decoupling is usually expected to be initiated by the break-down of annihilations. However, chemical decoupling can also be initiated by the break-down of conversion processes leading to conversion-driven freeze-out [7] (or co-scattering [8]). This latter possibility can be realized for very small DM-SM couplings.

In the Sec. 2 we review the general features of conversion-driven freeze-out. A realization within a simple $t$-channel mediator DM model, its numerical solutions and constraints are discussed in Sec. 3. We conclude in Sec. 4.

\footnotetext{
${ }^{1}$ For a viable exception see $e . g$. [5].
} 


\section{Conversion versus annihilations}

A commonly made assumption in co-annihilation scenarios is that conversion processes between DM and the slightly heavier co-annihilating partner(s) are thoroughly efficient during freezeout. In this case, relative chemical equilibrium is maintained, $n_{\chi_{i}} / n_{\chi_{i}}^{\mathrm{eq}}=n_{\chi_{j}} / n_{\chi_{j}}^{\mathrm{eq}}$, and annihilations can be described by an effective, thermally averaged cross section [6]

$$
\langle\sigma v\rangle_{\mathrm{eff}}=\sum_{i, j}\langle\sigma v\rangle_{i j} \frac{n_{\chi_{i}}^{\mathrm{eq}}}{n_{\chi}^{\mathrm{eq}}} \frac{n_{\chi_{j}}^{\mathrm{eq}}}{n_{\chi}^{\mathrm{eq}}},
$$

where $n_{\chi}^{\mathrm{eq}}=\sum_{i} n_{\chi_{i}}^{\mathrm{eq}}$ and $i, j$ runs over all dark sector particles.

For a large hierarchy in the couplings to the SM between DM and the co-annihilating partner(s), $\langle\sigma v\rangle_{i j}$ could be negligible for all channels containing DM in the initial state. Dark matter dilution is then entirely driven by annihilations of heavier states.

The rate of conversion processes, which contain light SM degrees of freedom in the initial state, is expected to be boosted relative to the rate of dark sector annihilations for $T<m_{\chi}$ :

$$
\frac{\Gamma_{\mathrm{con}}}{\Gamma_{\mathrm{ann}}} \sim \frac{n_{a}^{\mathrm{eq}}}{n_{\chi}^{\mathrm{eq}}} \frac{\langle\sigma v\rangle_{\chi_{i} a \rightarrow \chi_{j} b}}{\langle\sigma v\rangle_{\mathrm{eff}}} \sim \mathrm{e}^{m_{\chi} / T} \frac{\langle\sigma v\rangle_{\chi_{i} a \rightarrow \chi_{j} b}}{\langle\sigma v\rangle_{\mathrm{eff}}},
$$

where $a, b$ are SM particles. For simplicity, in (2.2) we only consider conversions via a $2 \rightarrow$ 2 scattering cross section. However, inverse decays or $2 \leftrightarrow 3$ scattering processes can also be important in general.

There are two regimes regarding the size of the conversion terms and hence the temperature where the chemical decoupling (indicated by the subscript $\mathrm{CD}$ ) in the dark sector takes place:

(i) $T_{\mathrm{CD}, \text { con }} \ll T_{\mathrm{CD} \text {, ann }}$ : Chemical equilibrium within the dark sector breaks down well after the chemical decoupling of the dark sector from the SM takes place. This resembles the usual co-annihilation scenario.

(ii) $T_{\mathrm{CD} \text {, con }} \gtrsim T_{\mathrm{CD} \text {, ann }}$ : Chemical decoupling of $\mathrm{DM}$ from the dark sector takes place before or during chemical decoupling of the dark sector from the SM. This regime is called conversiondriven freeze-out [7] (or co-scattering [8]).

In the latter case $\Gamma_{\text {con }}$ determines the relic density. For a typical freeze-out temperature of $T_{\mathrm{CD}}$, ann $\sim$ $m_{\chi} / 25$, and considering $\Gamma_{\text {con }} / \Gamma_{\text {ann }} \sim 1$ at chemical decoupling, $\langle\sigma v\rangle_{\chi_{i} a \rightarrow \chi_{j} b}$ could be smaller than $\langle\sigma \nu\rangle_{\text {eff }}$ by a factor of $\mathrm{e}^{-25} \sim 10^{-11}$. Accordingly, conversion-driven freeze-out is realized for very weak DM couplings to the SM.

\section{Numerical example}

\subsection{A minimal model}

We consider one of the simplest example models providing a co-annihillation dark sector, namely a simplified dark matter model with a $t$-channel mediator:

$$
\mathscr{L}_{\text {int }}=\left|D_{\mu} \tilde{q}\right|^{2}+\lambda_{\chi} \tilde{q} \bar{q} \frac{1-\gamma_{5}}{2} \chi+\text { h.c. },
$$


where $q$ is a SM quark field, $\tilde{q}$ is a scalar partner of the quark (sharing the same gauge quantum numbers) and the DM $\chi$ is a Majorana fermion. Here $D_{\mu}$ denotes the covariant derivative, $(1-$ $\left.\gamma_{5}\right) / 2$ is the left-handed projection operator and $\lambda_{\chi}$ is the DM coupling strength. The model is similar to a limiting case of supersymmetry where $\chi$ is a bino-like neutralino and $\tilde{q}$ is a right-handed squark. Unlike in the minimal supersymmetric SM, $\lambda_{\chi}$ is not set by the SM gauge couplings but considered to be a free parameter of the model.

The model respects a $Z_{2}$-symmetry under which all SM fields are even while $\chi$ and $\tilde{q}$ are odd. An additional Higgs portal coupling of type $\Phi^{\dagger} \Phi \tilde{q}^{\dagger} \tilde{q}$ is allowed by the symmetries as well. However, in the following we assume it to be negligible for simplicity since it does not affect the qualitative picture. For a more detailed discussion of the model and its phenomenology see for example $[7,9,10]$. We consider the two cases of a bottom and top partner, $q=b, t$.

\subsection{Evolution of abundances}

For the general case where neither $T_{\mathrm{CD} \text {, con }} \ll T_{\mathrm{CD} \text {, ann }}$ nor $T_{\mathrm{CD} \text {, con }} \gg T_{\mathrm{CD} \text {, ann }}$ is guaranteed a set of coupled Boltzmann equations has to be solved. For the minimal model considered here they read

$$
\begin{aligned}
\frac{\mathrm{d} Y_{\chi}}{\mathrm{d} x}= & \frac{1}{3 H} \frac{\mathrm{d} s}{\mathrm{~d} x}\left[\left\langle\sigma_{\chi \chi} v\right\rangle\left(Y_{\chi}^{2}-Y_{\chi}^{\mathrm{eq} 2}\right)+\left\langle\sigma_{\chi \tilde{q}} v\right\rangle\left(Y_{\chi} Y_{\tilde{q}}-Y_{\chi}^{\mathrm{eq}} Y_{\tilde{q}}^{\mathrm{eq}}\right)\right. \\
& +\frac{\Gamma_{\chi \rightarrow \tilde{q}}}{s}\left(Y_{\chi}-Y_{\tilde{q}} \frac{Y_{\chi}^{\mathrm{eq}}}{Y_{\tilde{q}}^{\mathrm{eq}}}\right)-\frac{\Gamma_{\tilde{q}}}{s}\left(Y_{\tilde{q}}-Y_{\chi} \frac{Y_{\tilde{q}}^{\mathrm{eq}}}{Y_{\chi}^{\mathrm{eq}}}\right)+\left\langle\sigma_{\chi \chi \rightarrow \tilde{q} \tilde{q}^{v}} v\left(Y_{\chi}^{2}-Y_{\tilde{q}}^{2} \frac{Y_{\chi}^{\mathrm{eq} 2}}{Y_{\tilde{q}}^{\mathrm{eq} 2}}\right)\right], \\
\frac{\mathrm{d} Y_{\tilde{q}}}{\mathrm{~d} x}= & \frac{1}{3 H} \frac{\mathrm{d} s}{\mathrm{~d} x}\left[\frac{1}{2}\left\langle\sigma_{\tilde{q} \tilde{q}^{\dagger}} v\right\rangle\left(Y_{\tilde{q}}^{2}-Y_{\tilde{q}}^{\mathrm{eq} 2}\right)+\left\langle\sigma_{\chi \tilde{q}} v\right\rangle\left(Y_{\chi} Y_{\tilde{q}}-Y_{\chi}^{\mathrm{eq}} Y_{\tilde{q}}^{\mathrm{eq}}\right)\right. \\
& -\frac{\Gamma_{\chi \rightarrow \tilde{q}}}{s}\left(Y_{\chi}-Y_{\tilde{q}} \frac{Y_{\chi}^{\mathrm{eq}}}{Y_{\tilde{q}}^{\mathrm{eq}}}\right)+\frac{\Gamma_{\tilde{q}}}{s}\left(Y_{\tilde{q}}-Y_{\chi} \frac{Y_{\tilde{q}}^{\mathrm{eq}}}{Y_{\chi}^{\mathrm{eq}}}\right)-\left\langle\sigma_{\chi \chi \rightarrow \tilde{q} \tilde{q}^{v}} v\left(Y_{\chi}^{2}-Y_{\tilde{q}}^{2} \frac{Y_{\chi}^{\mathrm{eq} 2}}{Y_{\tilde{q}}^{\mathrm{eq} 2}}\right)\right],
\end{aligned}
$$

where $Y_{i}=n_{i} / s$ is the comoving number density for the species $i, s$ is the entropy density, $H$ denotes the Hubble parameter while $x=m_{\chi} / T$ parametrizes the temperature dependence. The first two terms in brackets in each equation represent annihilations into SM particles, while the last three terms (second lines) represent conversions within the dark sector. Among these $\Gamma_{\chi \rightarrow \tilde{q}}$ is the thermally averaged rate for $2 \rightarrow 2$ (or $2 \leftrightarrow 3$, relevant for $q=t$ ) scattering, $\Gamma_{\tilde{q}}$ is the thermally averaged decay rate while the last term represents pair conversion within the dark sector. The latter can usually be neglected as it is subdominant $\left(\mathscr{O}\left(\lambda_{\chi}^{4}\right)\right)$ whenever chemical equilibrium is questionable (and hence $\lambda_{\chi}$ is small).

For sizeable couplings $\lambda_{\chi}$ the conversion rates are much faster than the Hubble rate such that chemical equilibrium in the dark sector is a good assumption. This can be used to reduce Eqs. (3.2) and (3.3) to a single equation in terms of $Y=\sum_{i} Y_{i}$ and the effective annihilation cross section defined in Eq. 2.1. ${ }^{2}$ This case certainly applies to supersymmetry where $\lambda_{\chi}=0.17(0.33)$ for co-annihilation with a right-handed sbottom (stop). The respective relative rates and abundances for the case of the sbottom are shown in the upper panels of Fig. 1 for the parameter point with $m_{\chi}=500 \mathrm{GeV}, m_{\widetilde{b}}=510 \mathrm{GeV}$. For this parameter point DM would be under-abundant.

\footnotetext{
${ }^{2}$ This is commonly done in numerical relic density solvers [11, 12, 13].
} 

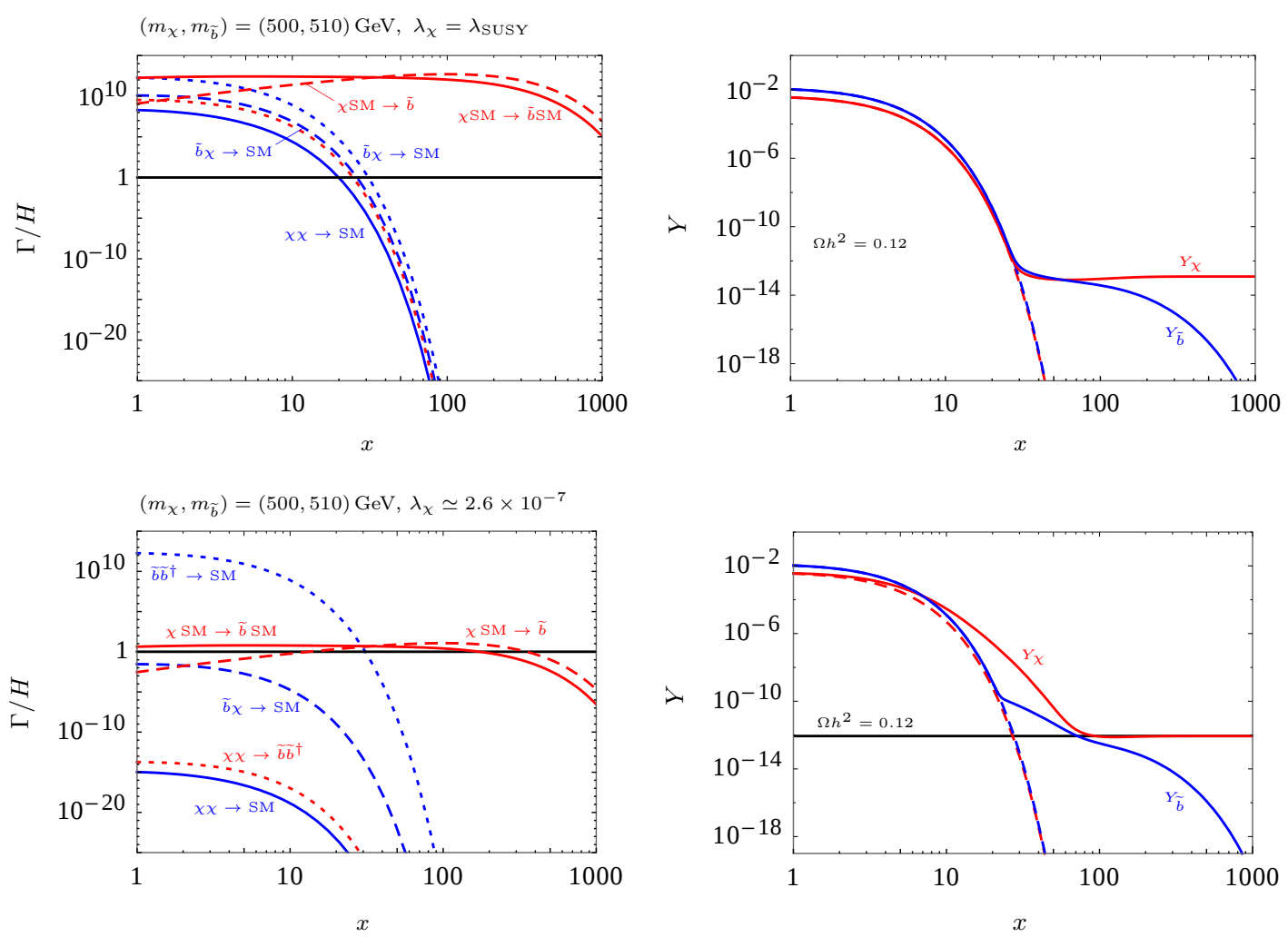

Figure 1: Left panels: Rates of annihilation (blue curves) and conversion (red curves) terms in the Boltzmann equation relative to the Hubble rate. Right panels: Evolution of the resulting abundance (solid curves) of $\widetilde{b}$ (blue) and $\chi$ (red) as well as the corresponding equilibrium abundances (dashed curves). Both quantities are plotted as a function of the temperature parameter $x=m_{\chi} / T$. We consider $m_{\chi}=500 \mathrm{GeV}, m_{\tilde{b}}=510 \mathrm{GeV}$ as well as $\lambda_{\chi}=\lambda_{\text {SUSY }} \simeq 0.17$ (upper panels) and $\lambda_{\chi} \approx 2.6 \times 10^{-7}$ (lower panels) [7].

For much smaller couplings, $\lambda_{\chi} \lesssim 10^{-6}$, conversion processes start to become inefficient during the freeze-out of $\tilde{b}, \Gamma_{\text {con }} / H \sim 1$. Therefore the solution of the coupled set of Boltzmann equations is necessary. ${ }^{3}$ The evolution of the relative rates and the abundance is shown for $\lambda_{\chi} \approx 2.6 \times 10^{-7}$ which is the coupling that provides the right relic density for the chosen mass point $m_{\chi}=500 \mathrm{GeV}, m_{\tilde{b}}=510 \mathrm{GeV}$. In this case $Y_{\chi}$ already departs from thermal equilibrium at around $x \sim 3$ while a significant depletion of DM occurs up to $x \simeq 100$. Both abundances have a non-trivial evolution. Hence, for this scenario the process of freeze-out extends over a large range of $x$ - in contrast to a typical WIMP.

Figure 2 shows the resulting relic density as a function of the coupling over the range from $10^{-7}$ to $\mathscr{O}(1)$. In the regime of $\mathscr{O}(1)$ couplings $\chi$ self-annihilation or $\chi-\tilde{b}$ co-annihilation are relevant and hence introduce a dependence on $\lambda_{\chi}$ (region A). For smaller couplings mediator

\footnotetext{
${ }^{3}$ As the elastic scattering cross section $\chi a \rightarrow \chi a$ is suppressed by $\lambda_{\chi}^{4}$, at first sight the assumption of kinetic equilibrium - justifying the solution of the integrated Boltzmann equations (3.2) and (3.3) - appears questionable as well. However, as shown explicitly in [7], the DM momentum distribution does not deviate significantly from the thermal one and the integrated equations provide a good approximation. Due to the small mass splitting DM approximately inherits the momentum distribution of the mediator, which is kept in kinetic equilibrium throughout the freeze-out process thanks to its strong coupling to the SM.
} 


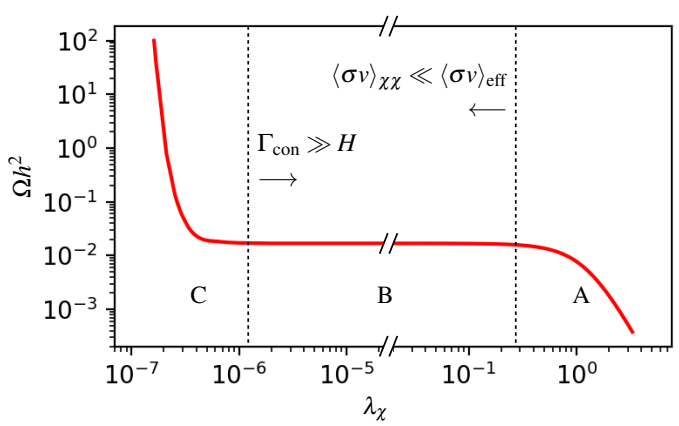

Figure 2: Relic density as a function of the DM coupling $\lambda_{\chi}$ for $m_{\chi}=500 \mathrm{GeV}, m_{\tilde{b}}=510 \mathrm{GeV}$. The regions $\mathrm{A}, \mathrm{B}$ and $\mathrm{C}$ denote the characteristic phenomenological regimes, see text for details.

pair-annihilations dominate the annihilation while $\lambda_{\chi}$ is still large enough to maintain chemical equilibrium in the dark sector, $\Gamma \gg H$ (region $\mathrm{B}$ ). In this region the relic density is independent of $\lambda_{\chi}$. Finally, for $\lambda_{\chi} \lesssim 10^{-6}$ we enter the conversion-driven regime (region $\mathrm{C}$ ), introducing a $\lambda_{\chi}$-dependence through the conversion rates.

\subsection{Viable parameter range}

Conversion-driven freeze-out opens up a cosmologically viable region in parameter space with small mass splittings between DM and the mediator that yields $\Omega h^{2} \simeq 0.12$. If chemical equilibrium was maintained within the dark sector, efficient co-annihilations would lead to $\Omega h^{2}<0.12$ in that case. The respective region in parameter space is shown in Fig. 3 below the thick black curve. In fact, the thick black curve separates the regions $\mathrm{A}$ (above) and $\mathrm{C}$ (below) defined in the last paragraph, while region B resides on the curve. Here we consider the case of the bottom- and top-philic DM model in the left and right panel, respectively.

The green thin curves in Fig. 3 indicate contours of constant coupling strength $\lambda_{\chi}$ providing $\Omega h^{2}=0.12$. The coupling varies between $10^{-7}$ and $10^{-6}$ in the bottom-philic model and $3 \times$ $10^{-6}$ and $10^{-3}$ for the top-philic model. The reason for the larger coupling and the larger range spanned in the latter case is due to the fact that all $2 \rightarrow 2$ conversion processes contain at least one heavy SM particle, namely a $W$-boson or a top-quark, in the initial or final state. This leads to an additional Boltzmann suppression (either directly through the $W$ or $t$ abundance or through phasespace suppression, if particles are in the initial or final state, respectively) which is particularly strong for small DM masses. In this regime $2 \leftrightarrow 3$ processes become dominant.

Figure 3 also displays the resulting lifetime (or decay length) of the mediator as (gray) dotted curves. The lifetime is drastically different for the two considered models. For the bottom-philic model, in a large portion of the allowed parameters space providing conversion-driven freeze-out the 2-body decay $\tilde{b} \rightarrow \chi b$ is open and hence the decay is only suppressed by the smallness of the coupling $\lambda_{\chi}$. In fact, for the bottom-philic model the decay rate and the $2 \rightarrow 2$ scattering rate during freeze-out are of the same order ( $c f$. left panels of Fig. 1). The resulting decay length is of the order of a few to a few tenth of a cm. This range provides interesting signatures at colliders, see Sec. 3.4.

The situation is vastly different in the top-philic model. In the allowed conversion-driven freeze-out parameter region the 2-body decay is forbidden and the decay of the mediator proceeds solely through the 4-body decay. Due to this extra suppression the decay length is always large 

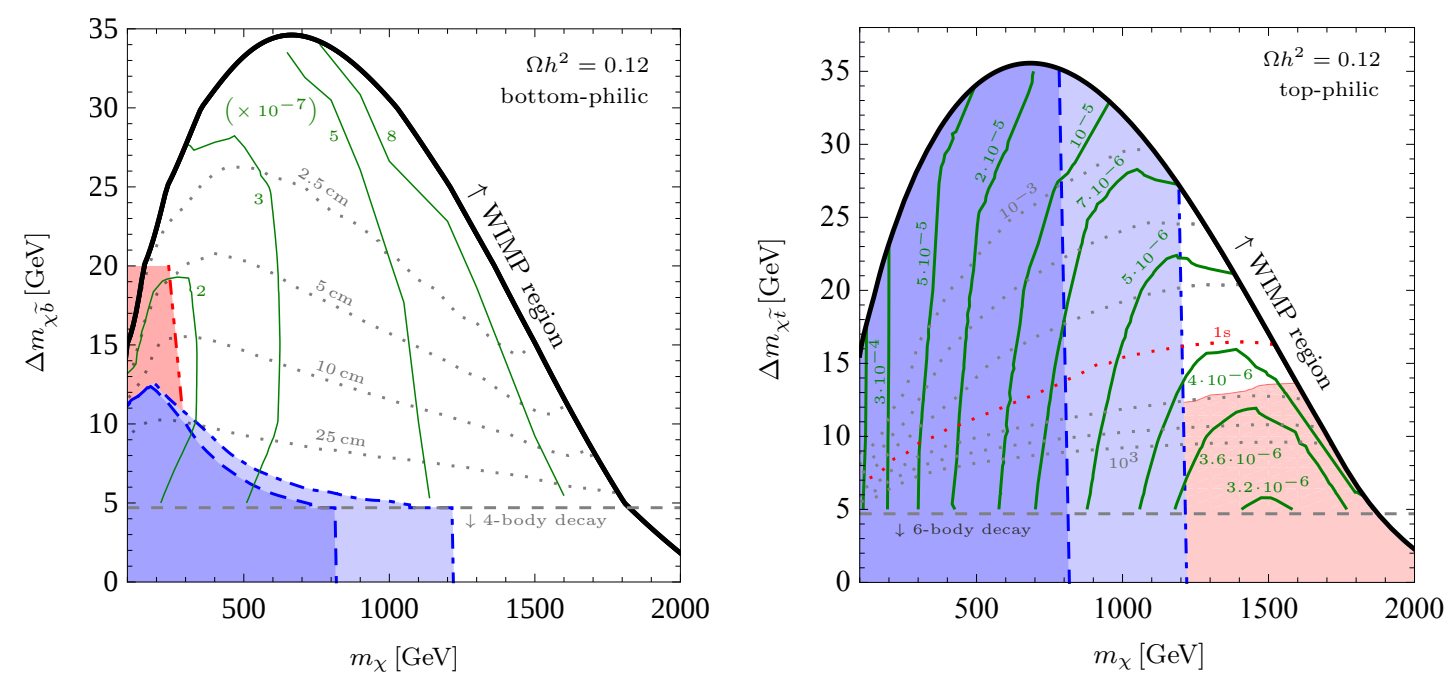

Figure 3: Cosmologically viable parameter space $\left(\Omega h^{2}=0.12\right)$ in the region providing conversion-driven freeze-out (below black thick curve) in the bottom- (left panel) and top-philic (right panel) model [7, 10]. We show the contours of constant $\lambda_{\chi}$ in green $\left(\times 10^{-7}\right.$ in the left panel). Contours of constant mediator decay length (left panel) and lifetimes (right panel) are shown as gray dotted curves. The latter is shown in the range $\left[10^{-3} ; 10^{3}\right] \mathrm{s}$ in steps of an order of magnitude (the curve for $1 \mathrm{~s}$ is highlighted in red). We show $95 \%$ C.L. exclusion regions from $R$-hadron searches at the 8 and $13 \mathrm{TeV}$ LHC in dark and light blue, respectively. The red shaded region bordered by the red dot-dot-dashed curve (left panel) denotes the constraint from mono-jet searches at the $13 \mathrm{TeV}$ LHC. The light red shaded region (right panel) indicates constraints from BBN. Below the horizontal gray dashed line $(\sim 5 \mathrm{GeV})$ the 2- (left panel) and 4-body decay (right panel) is kinematically forbidden rendering the 4- and 6-body decay, respectively, to be dominant.

compared to the detector-size of colliders. The long lifetime could even be in conflict with BBN for $\tau \gtrsim 1 \mathrm{sec}$. The respective bound utilizing the results from [14] is shown as the red shaded region in the right panel of Fig. 3. Furthermore, compared to the rates of the leading $2 \rightarrow 2$ or $2 \leftrightarrow 3$ conversion processes the decay rate is negligible during freeze-out.

\subsection{Distinct signature}

Due to the small couplings and the $\lambda_{\chi}^{4}$ suppression of the annihilation cross section $\chi \chi \rightarrow \mathrm{SM}$ or elastic DM-nucleon scattering $\chi \mathrm{SM} \rightarrow \chi \mathrm{SM}$ the allowed conversion-driven freeze-out region is not challenged by indirect or direct detection searches. However, the sizeable coupling required to efficiently annihilate away the mediator leads to significant interactions of the mediator with the SM and hence supports the possibility of searching for the mediator. In our example of a strongly coupled mediator its production cross section at the LHC is sizeable. On top of that the mediator decay length for conversion-driven freeze-out with mediator mass in the $\mathrm{GeV}-\mathrm{TeV}$ range is macroscopic. This leads to long-lived particles which constitute a very distinct signature at the LHC (for a recent, comprehensive account on long-lived particles at the LHC see [15]).

Defining $x_{\mathrm{dec}}$ to be the temperature parameter where the decay is on the edge of being efficient, $\Gamma_{\mathrm{dec}} \sim H\left(x_{\mathrm{dec}}\right)$, and assuming freeze-out to take place in the radiation dominated era with the 


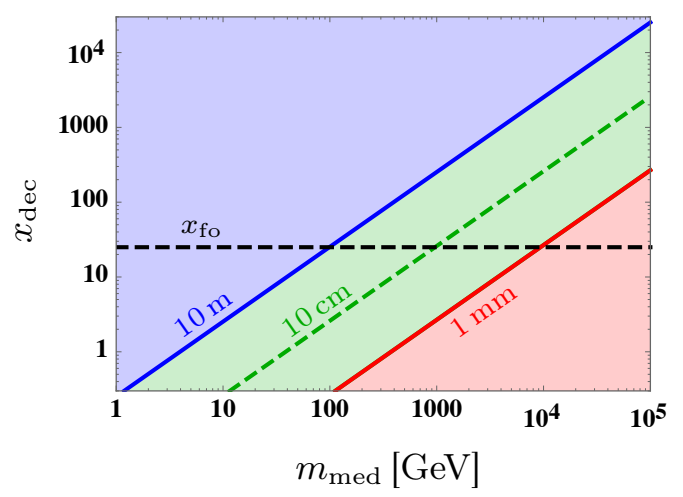

Figure 4: Regions of decay-length in the plane spanned by the mediator mass and the characteristic temperature parameter of its decay, $x_{\mathrm{dec}}$. The blue, green and red regions, respectively, indicate decays to typically take place outside the detector, within the sensitive detector volume or promptly. The dashed black horizontal line indicate a typical value for the temperature parameter at freeze-out, $x_{\mathrm{fo}}=25$.

number of relativistic degrees of freedom in the SM being $g_{*} \simeq 100$, we can derive

$$
c \tau \sim H^{-1}\left(x_{\mathrm{dec}}\right) \simeq 10 \mathrm{~cm}\left(\frac{1 \mathrm{TeV}}{m_{\mathrm{med}}}\right)^{2}\left(\frac{x_{\mathrm{dec}}}{25}\right)^{2}
$$

This general relation between the mass of the mediator and its decay-length, relevant for the LHC, is illustrated in Fig. 4. It highlights the domains of prompt decays (red shaded), decays within the detector (green shaded) and detector-stable mediators (blue shaded) in the plane spanned by the mediator mass and $x_{\mathrm{dec}}$. For the conversion-driven freeze-out scenario considered here $\Gamma_{\mathrm{con}} / H \sim 1$ around freeze-out, $x_{\mathrm{fo}} \sim 25$, and hence $x_{\mathrm{dec}} \sim x_{\mathrm{fo}}$ or $x_{\mathrm{dec}} \gg x_{\mathrm{fo}}$, depending on whether the decay provides a sizable contribution to the conversion or not. ${ }^{4}$ In our case the bottom- and top-philic model falls in the first and second class, respectively. For the mass range explorable at the current and future updates of the LHC the two scenarios predict lifetimes leading to decays inside the tracker and detector-stable mediators, respectively. Both signatures provide promising prospects.

The (reinterpreted) limits from the 8 and $13 \mathrm{TeV}$ LHC searches for heavy stable charged particles $[16,17]$ are shown as the dark and light blue shaded regions in Fig. 3. As the limits are suppressed due to the small fraction of mediators in the bottom-philic model that pass the whole detector (given its small decay length) these limits are much weaker in this case. In contrast the limits exclude a significant part of the allowed parameter space for the top-philic model leaving a small part of the parameter space between around 1.2 and $1.6 \mathrm{TeV}$ only which can be probed entirely by $300 \mathrm{fb}^{-1}$ at the 13 or $14 \mathrm{TeV}$ LHC [10].

\footnotetext{
${ }^{4}$ To be more precise, in the former case $\Gamma_{\mathrm{dec}} / H \sim 1$ should rather hold for the rate of the inverse decay which behaves like $\Gamma_{\text {dec, inv }}=n_{\tilde{q}}^{\mathrm{eq}} / n_{\chi}^{\mathrm{eq}} \Gamma_{\mathrm{dec}} \sim \mathrm{e}^{-\Delta m / T} \Gamma_{\mathrm{dec}}$. In contrast to the decay which becomes more and more efficient with decreasing temperature ( $\Gamma_{\operatorname{dec}} / H \propto T^{-2}$ in the non-relativistic regime and in radiation domination), the inverse decay experiences an additional exponential suppression when $T \lesssim \Delta m$. It hence reaches its maximum before this suppression becomes sizeable. Therefore, by construction, in scenarios where the inverse decay is a relevant contribution to conversion, chemical decoupling takes places at $T \sim \Delta m$ where the size of the decay and inverse decay rate is of the same order of magnitude. Nevertheless, we emphasize that, due to a number of neglected $\mathscr{O}(1)$-factors, eq. (3.4) only provides a rough order-of-magnitude estimate.
} 
Another existing search probing a small part of the allowed parameter region of the bottomphilic model is the mono-jet search [18] that solely relies on the recoil against the missing energy caused by DM, not exploiting the macroscopic decay length of the mediator. The corresponding limit is shown as the red shaded region in the left panel of Fig. 3. A dedicated search for disappearing $R$-hadron tracks or displaced jets (kinked tracks) is expected to greatly strengthen the constraints on the model covering large parts of the yet unexplored region. Current searches e.g. for disappearing tracks from long-lived charginos are not directly applicable to our scenario.

\section{Conclusion}

In this article we discussed a novel variant of freeze-out that we call conversion-driven freezeout. In this scenario DM interacts only very weakly with the SM rendering its self-annihilation negligible during freeze-out. Nevertheless, a sufficient dilution and cooling of DM can be driven by a mediator with strong thermal contact to the SM in combination with conversion processes within the dark sector. Typically, the latter require much smaller couplings to be efficient than annihilation processes during chemical decoupling due to the appearance of light SM particles in the initial state with non-Boltzmann suppressed number densities. However, for sufficiently small coupling they can initiate the chemical decoupling and hence govern the relic density. This scenario provides a distinct phenomenology.

In general, the relic density computation in this scenario requires the numerical solution of the full coupled set of Boltzmann equations. We discuss a simple $t$-channel model with a scalar top or bottom partner as a mediator. Both choices provide a cosmologically valid parameter space in the conversion-driven regime with couplings in the range between $10^{-7}$ and $10^{-3}$ - out of reach of indirect or direct detection searches in the foreseeable future. However, due to a sizeable production cross section at colliders and macroscopic decay length of the mediator, the scenario can be probed by long-lived particle searches at the LHC. While the former model predicts detector-stable mediators the latter predicts mediator decay length of the order of the tracker size providing disappearing $R$-hadron tracks and/or displaced $b$-jets.

\section{Acknowledgements}

J.H. acknowledges support from the F.R.S.-FNRS, of which he is a postdoctoral researcher as well as support by the German Academic Exchange Service (DAAD) through the travel grant "Kongressreisen 2018". M.H. acknowledges supported by the ERC Starting Grant 'NewAve' (638528) as well as by the Deutsche Forschungsgemeinschaft (DFG, German Research Foundation) under Germany’s Excellence Strategy (EXC 2121) 'Quantum Universe' (390833306).

\section{References}

[1] G. Bertone, D. Hooper and J. Silk, Particle dark matter: Evidence, candidates and constraints, Phys. Rept. 405 (2005) 279-390, [hep-ph / 0404175$].$

[2] PlancK collaboration, P. A. R. Ade et al., Planck 2015 results. XIII. Cosmological parameters, Astron. Astrophys. 594 (2016) A13, [1502.01589]. 
[3] FERMI-LAT, DES collaboration, A. Drlica-Wagner et al., Search for Gamma-Ray Emission from DES Dwarf Spheroidal Galaxy Candidates with Fermi-LAT Data, Astrophys. J. 809 (2015) L4, [1503.02632].

[4] A. Cuoco, J. Heisig, M. Korsmeier and M. Krämer, Constraining heavy dark matter with cosmic-ray antiprotons, JCAP 1804 (2018) 004, [1711.05274].

[5] R. T. D'Agnolo, C. Mondino, J. T. Ruderman and P.-J. Wang, Exponentially Light Dark Matter from Coannihilation, JHEP 08 (2018) 079, [1803.02901].

[6] J. Edsjo and P. Gondolo, Neutralino relic density including coannihilations, Phys. Rev. D56 (1997) 1879-1894, [hep-ph/9704361].

[7] M. Garny, J. Heisig, B. Lülf and S. Vogl, Coannihilation without chemical equilibrium, Phys. Rev. D96 (2017) 103521, [1705.09292].

[8] R. T. D’Agnolo, D. Pappadopulo and J. T. Ruderman, Fourth Exception in the Calculation of Relic Abundances, Phys. Rev. Lett. 119 (2017) 061102, [1705.08450].

[9] A. Ibarra, A. Pierce, N. R. Shah and S. Vogl, Anatomy of Coannihilation with a Scalar Top Partner, Phys. Rev. D91 (2015) 095018, [1501.03164].

[10] M. Garny, J. Heisig, M. Hufnagel and B. Lülf, Top-philic dark matter within and beyond the WIMP paradigm, Phys. Rev. D97 (2018) 075002, [1802.00814].

[11] G. Bélanger, F. Boudjema, A. Goudelis, A. Pukhov and B. Zaldivar, micrOMEGAs5.0 : Freeze-in, Comput. Phys. Commun. 231 (2018) 173-186, [1801.03509].

[12] F. Ambrogi, C. Arina, M. Backovic, J. Heisig, F. Maltoni, L. Mantani et al., MadDM v.3.0: a Comprehensive Tool for Dark Matter Studies, Phys. Dark Univ. 24 (2019) 100249, [1804. 00044 ].

[13] T. Bringmann, J. Edsjö, P. Gondolo, P. Ullio and L. Bergström, DarkSUSY 6 : An Advanced Tool to Compute Dark Matter Properties Numerically, JCAP 1807 (2018) 033, [1802 . 03399 ].

[14] K. Jedamzik, Big bang nucleosynthesis constraints on hadronically and electromagnetically decaying relic neutral particles, Phys. Rev. D74 (2006) 103509, [hep-ph/0604251].

[15] J. Alimena et al., Searching for long-lived particles beyond the Standard Model at the Large Hadron Collider, 1903.04497.

[16] CMS collaboration, V. Khachatryan et al., Constraints on the pMSSM, AMSB model and on other models from the search for long-lived charged particles in proton-proton collisions at sqrt $(\mathrm{s})=8 \mathrm{TeV}$, Eur. Phys. J. C75 (2015) 325, [1502 .02522].

[17] CMS collaboration, V. Khachatryan et al. Tech. Rep. CMS-PAS-EXO-16-036, 2016.

[18] ATLAS collaboration, M. Aaboud et al., Search for new phenomena in final states with an energetic jet and large missing transverse momentum in pp collisions at $13 \mathrm{TeV}$ using the ATLAS detector, Phys. Rev. D94 (2016) 032005, [1604.07773]. 